\title{
Optical Electrostriction
}

\author{
Richard G. Crisp and David L. Andrews \\ Nanostructures and Photomolecular Systems, School of Chemical Sciences, University of East \\ Anglia, Norwich, NR4 7TJ, U.K.
}

\begin{abstract}
It is well known that the forces which light imparts on micro- and nanoparticles arise due to intensity gradients and dielectric mismatch. For laser-irradiated atoms and molecules, optical forces primarily result from close resonance between the optical frequency and an electronic transition. Recently it has emerged that optically induced pair forces also arise, through a modification of Casimir-Polder interactions; preliminary assessments of the mechanism have largely centered on nanoparticle systems. In this paper, we show that a potentially very significant effect can be anticipated in the condensed phase, an optically induced modification of interatomic forces that is capable of generating anisotropic patterns of laser-induced compression and expansion. This phenomenon, termed optical electrostriction, should be measurable and significant when high intensity laser light is transmitted through even an essentially nonabsorptive material. However, the full conditions for observation of the effect are such that some competing interactions might also arise. Key parameters that determine the size and character of optical electrostriction are delineated and possible applications are considered, including optical actuators for nanoscale electromechanical systems.
\end{abstract}

Keywords: Optomechanical forces, electrostriction, laser optics, Casimir-Polder interaction, quantum electrodynamics, nanoscale electromechanical systems

\section{INTRODUCTION}

The use of laser light to manipulate and guide small particles is an increasingly prominent research tool, finding applications in diverse laboratories. A variety of optomechanical mechanisms operate, across a spectrum of techniques that ranges from optical tweezers to laser cooling and trapping, though all are based on forces that operate directly on individual particles. Recently a wave of excitement has been created by a discovery of an entirely different class of optically induced forces, which operate between particles, over nanoscale dimensions. ${ }^{1-10}$ Such forces offer a number of highly distinctive features which can be exploited for the controlled optical manipulation of matter. Through such interactions, new opportunities for creating optically ordered matter have already been demonstrated both theoretically and experimentally, leading to the introduction of terms such as 'optical binding' and 'optical matter' in the recent literature. ${ }^{11-13}$ Though slightly misleading, these are terms that rightly draw attention to new, intriguing and distinctive phenomena.

The first demonstration of optically induced inter-particle forces occurred in $1980^{14}$, utilizing the theory of quantum electrodynamics (QED). This was followed by sporadic investigations involving different methods. ${ }^{15-20}$ The advent of sufficient laser intensities to study these forces are now routinely available, encouraging new interest; indeed, there is now good reason to expect significant effects to be experimentally demonstrable at much lower intensity levels than originally expected. In 2005 the UEA quantum electrodynamics group published the first fully comprehensive theory of optically induced inter-particle force, based on QED. ${ }^{21}$ Specific calculations were also performed for carbon nanotubes, ${ }^{22}$ and the practicality of measuring significant laser-induced forces and torques was proven. It was also shown that the use of wavefront-structured light, such as Laguerre-Gaussian beams, offers further scope to tailor the pattern of such forces and torques; it can, for example, provide a means of achieving particle ring formations. ${ }^{23}$

In recently published material, ${ }^{24}$ we have shown how the mechanism for optical binding owes its origin to a modification of Casimir-Polder forces. One of the possible manifestations that we have recently begun to consider is the effect of intense throughput optical radiation on optically transparent solids, where it is conceivable that effects 
extending to the microscale might arise from the combined influence of light on the nearest-neighbor interactions between constituent particles of matter (atoms or molecules). It is the further exploration and quantification of this solid-state effect that we report below, as well as possible mechanisms for the detection of associated mechanical forces.

\section{PERTURBATIVE CALCULATIONS}

We begin by defining the components of a simple system comprising a pair of particles A and B, each with distinct electronic integrity and electrical neutrality, together with the radiation field. As is appropriate for the subsequent applications detailed specifically, the term 'molecules' will be used in the following as a generic descriptor of these particles. Representing the system in quantum electrodynamical terms in the Coulomb gauge ensures that the coupling fields are duly retarded and satisfy causality. ${ }^{25}$ In multipolar form the system Hamiltonian may be represented thus;

$$
H=\sum_{\xi=\mathrm{A}, \mathrm{B}} H_{\mathrm{mol}}^{\xi}+\sum_{\xi=\mathrm{A}, \mathrm{B}} H_{\mathrm{int}}^{\xi}+H_{\mathrm{rad}},
$$

Here $H_{\mathrm{mol}}^{\xi}$ is the field-free multipolar Hamiltonian for molecule $\xi$, operator $H_{\mathrm{int}}^{\xi}$ represents the interaction of $\xi$ with the radiation field, and $H_{\text {rad }}$ is the radiation Hamiltonian. The tripartite simplicity of equation (2.1) specifically results from adoption of the multipolar form of light-matter interaction, based on a well-known canonical transformation from the minimal-coupling interaction. ${ }^{26-28}$ This procedure results in a precise cancellation from the system Hamiltonian of all Coulombic terms, save those intrinsic to the internal structure of the Hamiltonian operators for the component molecules. In the electric dipole approximation, $H_{\mathrm{int}}^{\xi}$ is given by;

$$
H_{\mathrm{int}}^{\xi}=-\varepsilon_{0}^{-1} \sum_{\xi} \mu(\xi) \cdot \mathbf{d}^{\perp}\left(\mathbf{R}_{\xi}\right)
$$

with $\mu(\xi)$ and $\mathbf{R}_{\xi}$ respectively denoting the electric-dipole moment operator and the position vector of molecule $\xi$. The operator $\mathbf{d}^{\perp}\left(\mathbf{R}_{\xi}\right)$, representing the transverse electric displacement field at that location, is expressible in the following mode expansion involving summations over optical wave-vectors, $\mathbf{p}$, and polarizations, $\varepsilon$,

$$
\mathbf{d}^{\perp}\left(\mathbf{R}_{\xi}\right)=\mathrm{i} \sum_{\mathbf{p}, \varepsilon}\left(\frac{\hbar c p \varepsilon_{0}}{2 V}\right)^{1 / 2}\left\{\mathbf{e}^{(\varepsilon)}(\mathbf{p}) a^{(\varepsilon)}(\mathbf{p}) e^{\mathrm{i}\left(\mathbf{p} \cdot \mathbf{R}_{\xi}\right)}-\overline{\mathbf{e}}^{(\varepsilon)}(\mathbf{p}) a^{\dagger(\varepsilon)}(\mathbf{p}) e^{-\mathrm{i}\left(\mathbf{p} \cdot \mathbf{R}_{\xi}\right)}\right\}
$$

Here $\mathbf{e}^{(\varepsilon)}(\mathbf{p})$ is the polarization unit vector $\left(\overline{\mathbf{e}}^{(\varepsilon)}(\mathbf{p})\right.$ being its complex conjugate, the admission of complex polarizations allowing for circular or elliptical as well as plane polarization); $V$ is an arbitrary quantization volume and $a^{(\varepsilon)}(\mathbf{p}), a^{\dagger(\varepsilon)}(\mathbf{p})$ are respectively the photon annihilation and creation operators for a radiation mode $(\mathbf{p}, \varepsilon)$.

To secure a general result for the optically induced energy-shift, $\Delta E$, and hence the associated force between $\mathrm{A}$ and $\mathrm{B}$, requires the implementation of fourth-order perturbation theory - since the interaction entails four moleculeradiation field coupling events;

$$
\Delta E=\operatorname{Re}\left\{\sum_{t, s, r} \frac{\left\langle i\left|H_{\text {int }}\right| t\right\rangle\left\langle t\left|H_{\text {int }}\right| s\right\rangle\left\langle s\left|H_{\text {int }}\right| r\right\rangle\left\langle r\left|H_{\text {int }}\right| i\right\rangle}{\left(E_{i}-E_{t}\right)\left(E_{i}-E_{s}\right)\left(E_{i}-E_{r}\right)}\right\} .
$$

Here all states are those of the system, i.e. the two molecules plus the radiation field; $|i\rangle$ is the unperturbed system state in which both molecules are in their electronic ground state, $|r\rangle,|s\rangle$ and $|t\rangle$ are virtual states, and $E_{n}$ is the energy of state $|n\rangle$. The latter signifies one of the basis states for the perturbative development, expressible in the form; 


$$
|n\rangle=\left|\operatorname{mol}_{n}\right\rangle\left|\operatorname{rad}_{n}\right\rangle \equiv\left|\operatorname{mol}_{n} ; \operatorname{rad}_{n}\right\rangle
$$

with $\left|\operatorname{mol}_{n}\right\rangle$ and $\left|\operatorname{rad}_{n}\right\rangle$ defining states of the molecular pair and the radiation, respectively. In equation (2.4), each operation of $H_{\text {int }}$ on the state to its right effects the annihilation or creation of a photon, as follows from (2.2) and (2.3). The laser-induced interaction involves the annihilation of a throughput photon at one molecule and the stimulated emission of an equivalent 'real' photon into the radiation mode; this is mediated by inter-molecular energy transfer through a virtual photon created at one site and annihilated at the other. The molecules and the throughput radiation suffer no overall change in state; the term 'real' applied to photons of the input mode denotes quanta of electromagnetic radiation with a propagation time that is long compared to the optical cycle, and correspondingly 'real' characteristics. ${ }^{29}$ In performing energy shift calculations based on (2.4), detailed representations of all contributory terms are provided by a set of 48 time-ordered diagrams, one of which is exhibited in figure 1.

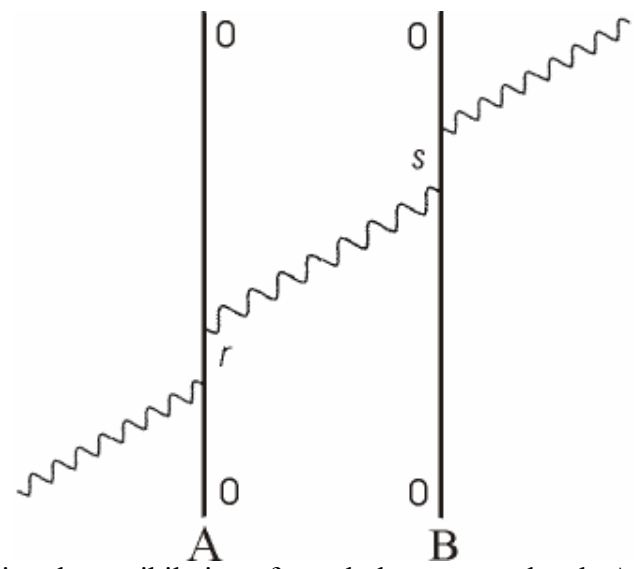

Figure 1: Time-ordered diagram depicting the annihilation of a real photon at molecule $\mathrm{A}$ and the creation of a corresponding real photon at molecule B, coupled by the transfer of a virtual photon between the pair. This diagram is one of 48 generated by eqn. 2.4

When such a large number of time-orderings is involved, a recently devised alternative based on state-sequence diagrams ${ }^{30}$ proves advantageous. All the time-orderings are in fact accommodated in just two state-sequence diagrams, (one for the case where the real photon absorption occurs at A and the stimulated emission at B, the other where the opposite applies), one of which is shown in figure 2.

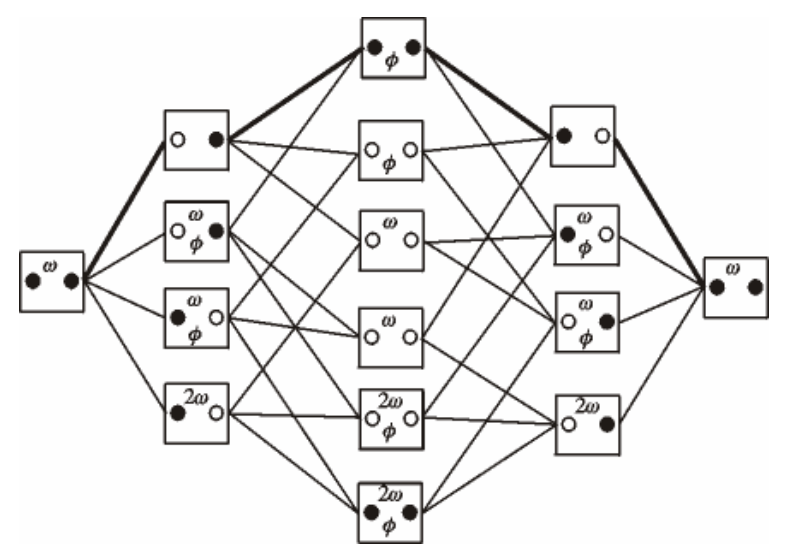

Figure 2: State-sequence diagram containing 24 of the time-ordered diagrams generated by eqn. 2.4. Figure 1 is incorporated as the highlighted pathway. Filled circles represent molecules in the ground state and empty circles virtual transition states. The symbols $\omega$ and $\phi$ represent real and virtual photons, respectively. 
The explicit result for $\Delta E$ follows the substitution of equations (2.2) and (2.3) in (2.4), recognizing that $\mu(\xi)$ and $\mathbf{d}^{\perp}\left(\mathbf{R}_{\xi}\right)$ operate on $\left|\operatorname{mol}_{n}\right\rangle$ and $\left|\operatorname{rad}_{n}\right\rangle$, respectively - the latter through the following expressions: $a^{(\varepsilon)}(\mathbf{p})|n(\mathbf{p}, \varepsilon)\rangle=\sqrt{n}|(n-1)(\mathbf{p}, \varepsilon)\rangle \quad$ and $\quad a^{\dagger(\varepsilon)}(\mathbf{p})|n(\mathbf{p}, \varepsilon)\rangle=\sqrt{n+1}|(n+1)(\mathbf{p}, \varepsilon)\rangle . \quad$ Details of the complete calculation are given in a recent paper. ${ }^{21}$ The following result thereby emerges, concisely expressible using the implied summation convention for repeated Cartesian tensor indices;

$$
\Delta E(k, \mathbf{R})=\left(\frac{n \hbar c k}{\varepsilon_{0} V}\right) \operatorname{Re}\left\{e_{i}^{(\lambda)} \bar{e}_{l}^{(\lambda)} \alpha_{i j}^{\mathrm{A}}(k) V_{j k}^{ \pm}(k, \mathbf{R}) \alpha_{k l}^{\mathrm{B}}(k) \exp (\mathrm{ik} \mathbf{R} \mathbf{R})\right\} .
$$

Here $\mathbf{k}$ and $\hbar c k$ denote the input wave-vector and photon energy, respectively, and $\mathbf{R}$ is the inter-particle displacement vector, $\mathbf{R} \equiv \mathbf{R}_{B}-\mathbf{R}_{A}$. Also $V_{j k}^{ \pm}$signifies the fully retarded resonance electric dipole - electric dipole interaction tensor of the form; ${ }^{31}$

$$
V_{j k}^{ \pm}(k, \mathbf{R})=\frac{e^{\mathrm{F} i k R}}{4 \pi \varepsilon_{0} R^{3}}\left\{(1 \pm \mathrm{i} k R)\left(\delta_{j k}-3 \hat{R}_{j} \hat{R}_{k}\right)-(k R)^{2}\left(\delta_{j k}-\hat{R}_{j} \hat{R}_{k}\right)\right\}
$$

and $\alpha_{i j}^{\xi}(k)$ is the dynamic polarizability tensor given as;

$$
\alpha_{i j}^{\xi}(k)=\sum_{s}\left\{\frac{\mu_{i}^{i s} \mu_{j}^{s i}}{\tilde{E}_{s i}-\hbar c k}+\frac{\mu_{j}^{i s} \mu_{i}^{s i}}{\tilde{E}_{s i}+\hbar c k}\right\}
$$

where $\boldsymbol{\mu}^{x y}=\langle x|\boldsymbol{\mu}| y\rangle$ and $\tilde{E}_{x y} \equiv \tilde{E}_{x}-\tilde{E}_{y}$, the tilde denoting the inclusion of damping factors as appropriate.

Before proceeding further, we note that the above result in principle holds for two particles in a vacuum, each with properties expressible in terms of wavefunctions that extend over the whole particle - as is essentially the case for the molecules of a gas. In the condensed phase - the case of molecular solids, or for example particles optically trapped in a host liquid, dissipative and refractive corrections due to the electronic properties of the local environment should be applied to both the inter-particle coupling (which is thereby cast in terms of virtual polaritons rather than photons) and also the interactions with the optical beam. The procedure for introducing such corrections is intricate but the outcome is known $\mathrm{w}^{32,33}$ and the necessary reformulation of the above result produces the following equation:

$$
\Delta E(k, \mathbf{R})=\operatorname{Re}\left(\frac{n \varepsilon_{0} \hbar v_{k} k}{n_{k}^{3} V}\right)\left(\frac{n_{k}^{2}+2}{3}\right)\left\{e_{i}^{(\lambda)} \bar{e}_{l}^{(\lambda)} \chi_{i j}^{\prime \mathrm{A}}(k) V_{j k}^{ \pm}\left(n_{k} k, \mathbf{R}\right) \chi_{k l}^{\prime \mathrm{B}}(k) \exp (\mathrm{ik} . \mathbf{R})\right\}
$$

where $\chi^{\prime}$ denotes a linear susceptibility tensor scaled by the corresponding particle volume, $v_{k}$ is the group velocity for the medium at optical frequency $c k$, and $n_{k}$ is the corresponding complex refractive index. Although we do not pursue it here, it could be a matter of considerable interest to further develop this equation for nanoparticles such as quantum dots with distinctive dispersion characteristics.

\section{APPLICATIONS}

In order to observe meaningful shifts in inter-particle forces, a basic requirement is pairs of highly polarizable particles in close proximity to each other, with suitably intense laser radiation present. In previous publications we have focused on interactions between nanoparticles, in one case specifically addressing carbon nanotubes. ${ }^{22}$ The latter are particularly 
well suited as candidates for observing and exploiting laser-induced pair forces, because of their exceptional electronic properties. At first, it may appear unlikely that similar effects will be observed in smaller molecules due to their reduced polarizability. Two possible approaches can be taken to overcome this problem - either choose a system that is amenable to extremely high resolution mechanical or spectroscopic analysis, so that very small geometric adaptations to an optical pair force can be determined, or probe a system in which small mechanical effects are amplified by scale. In the following we entertain detailed examples of these two distinct cases.

\subsection{Van der Waals molecules}

Van der Waals molecules are weakly bound, usually dimeric molecular structures. Having significantly larger moments of inertia than their monomer parents - this difference enhanced by the unusually long 'bond' holding the component units together - such dimers are readily identifiable by high resolution microwave spectroscopy. The model system to be examined in more detail below consists of two linear molecules lying end-to-end, with the intermolecular separation vector $\mathbf{R}$ identified with the $\mathrm{Z}$-axis and the plane-polarized throughput radiation defined by $\phi$ i.e. the angle between the $\mathbf{e}$ and $\mathbf{R}$ vectors. The polarization vector can thus be written in cylindrical form as $\mathbf{e}=\sin \phi \hat{\mathbf{i}}+\cos \phi \hat{\mathbf{k}}$. From eqn (2.6), and acknowledging that $I(k)=n \hbar c^{2} k / V$ is the irradiance of the throughput radiation, the energy shift is;

$$
\begin{aligned}
& \Delta E(R)=\frac{I}{\varepsilon_{0} c} \operatorname{Re}\left\{\sum_{J, K} \sin ^{2} \phi \cdot \alpha_{X J}^{A} V_{J K}^{ \pm} \alpha_{K X}^{B}+\sin \phi \cos \phi\left(\alpha_{X J}^{A} V_{J K}^{ \pm} \alpha_{K Z}^{B}+\alpha_{Z J}^{A} V_{J K}^{ \pm} \alpha_{K X}^{B}\right)\right. \\
& \left.\quad+\cos ^{2} \phi \cdot \alpha_{Z J}^{A} V_{J K}^{ \pm} \alpha_{K Z}^{B}\right\} \cos (\mathbf{k} \cdot \mathbf{R})
\end{aligned}
$$

where the $k$ and $\mathbf{R}$ dependences are henceforth suppressed and the indices $I, J$ and $K$ are in the laboratory frame. Employing the explicit form of the $V_{J K}^{ \pm}$tensor from (2.7), and writing $\alpha_{X X}=\alpha_{\perp}, \alpha_{Z Z}=\alpha_{\|}$for each molecule, equation (3.1) is expressible as;

$$
\begin{aligned}
\Delta E(R)=\frac{I}{4 \pi \varepsilon_{0}^{2} c} & {\left[\left(\sin ^{2} \phi \cdot \alpha_{\perp}^{A} \alpha_{\perp}^{B}-2 \cos ^{2} \phi \cdot \alpha_{\|}^{A} \alpha_{\|}^{B}\right) \cdot\left(\frac{\cos k R}{R^{3}}+\frac{k \sin k R}{R^{2}}\right)\right.} \\
& \left.-\sin ^{2} \phi \cdot \alpha_{\perp}^{A} \alpha_{\perp}^{B}\left(\frac{k^{2} \cos k R}{R}\right)\right] \cos (\mathbf{k} \cdot \mathbf{R})
\end{aligned}
$$

In the short-range region $(k R<<1)$ the leading term of equation (3.2), $\Delta E^{0}$, is found by taking the leading terms in the Taylor series expansions of $\sin k R, \cos k R$ and $\cos (\mathbf{k} . \mathbf{R})$ to give;

$$
\Delta E^{0}(R)=\frac{I}{4 \pi \varepsilon_{0}^{2} c R^{3}}\left(\sin ^{2} \phi \cdot \alpha_{\perp}^{A} \alpha_{\perp}^{B}-2 \cos ^{2} \phi \cdot \alpha_{\|}^{A} \alpha_{\|}^{B}\right)
$$

On isotropically averaging the system with respect to the incoming light, the energy-shift of equation (3.3) is written as;

$$
\Delta E^{0}(R)=\frac{I}{6 \pi \varepsilon_{0}^{2} c R^{3}}\left(\alpha_{\perp}^{A} \alpha_{\perp}^{B}-\alpha_{\|}^{A} \alpha_{\|}^{B}\right)
$$

The result (3.4) vanishes if $\alpha_{\perp}^{A} \alpha_{\perp}^{B}=\alpha_{\|}^{A} \alpha_{\|}^{B}$ (i.e. if the molecules are spherically symmetric); otherwise it is non-zero and its sign signifies a force that is either attractive or repulsive, as determined by the relative magnitudes of the polarizability components. 
The van der Waals dimer $(\mathrm{HCN})_{2}$ is one widely-known example of such a molecular system. The intermolecular bond is well modeled by the Stockmayer potential; ${ }^{34,35}$

$$
\begin{aligned}
U\left(\mathbf{R}, \boldsymbol{\mu}^{A}, \boldsymbol{\mu}^{B}\right)= & 4 \varepsilon\left[(\sigma / R)^{12}-(\sigma / R)^{6}\right]+\boldsymbol{\mu}^{A} \cdot \boldsymbol{\mu}^{B} / 4 \pi \varepsilon_{0} R^{3}, \\
& -3\left(\boldsymbol{\mu}^{A} \cdot \mathbf{R}\right)\left(\boldsymbol{\mu}^{B} \cdot \mathbf{R}\right) / 4 \pi \varepsilon_{0} R^{5},
\end{aligned}
$$

where $R$ is the intermolecular separation, $\sigma$ is the usual Lennard-Jones parameter, $\varepsilon$ is the well-depth and the $\mu$ terms are dipole moments. The effect of intense throughput laser radiation is to introduce a laser-induced energy shift which can be included in an effective potential, writing;

$$
U(R)+\Delta E^{0}(R)=U(R)+K / R^{3}
$$

where, from eqn (3.4), $K=I\left(\alpha_{\perp}^{A} \alpha_{\perp}^{B}-\alpha_{\|}^{A} \alpha_{\|}^{B}\right) / 6 \pi \varepsilon_{0}^{2} c$. The modification to the potential energy surface changes the equilibrium position of the bond, relating to a contraction or expansion - depending on the attractive or repulsive nature, respectively, of the laser-induced energy shift. Differentiating eqn (3.6) with respect to $R$ gives the following at the new equilibrium position $R_{0}^{\prime}=R_{0}+\delta R_{0}$;

$$
U^{\prime}\left(R_{0}+\delta R_{0}\right)-3 K /\left(R_{0}+\delta R_{0}\right)^{4}=0
$$

Taking the leading term in Taylor series expansions of both terms in the above equation leads to an expression for $\delta R_{0}$ as follows;

$$
\delta R_{0}=\frac{3 K}{R_{0}^{4} U^{\prime \prime}\left(R_{0}\right)}
$$

Differentiating eqn (3.5) twice with respect to $R$ gives the following expression for the change in equilibrium bond length;

$$
\delta R_{0}=\frac{\pi I(k)\left(\alpha_{\perp}^{\prime 2}-\alpha_{\|}^{\prime 2}\right) R / 9 c}{\varepsilon\left[26 \sigma^{12} / R^{9}-7 \sigma^{6} / R^{3}\right]-|\mu|^{2} / 4 \pi \varepsilon_{0}},
$$

recognizing that $\alpha^{\mathrm{A}}=\alpha^{\mathrm{B}}=\alpha$ and $\mu^{\mathrm{A}}=\mu^{\mathrm{B}}=\mu$. In the above, $\alpha^{\prime}$ refers to volume polarizabilities $\left(\alpha\right.$ scaled by $\left.1 / 4 \pi \varepsilon_{0}\right)$.

The $(\mathrm{HCN})_{2}$ dimer is a particularly suitable example of a van der Waals molecule. Its centre of mass lies 0.605 $\AA$ from the central $\mathrm{H}$ atom, along the intermolecular bond. From the moment of inertia $I$ about the centre of mass, the rotational constant $B$ of the dimer can be calculated from;

$$
B=h / 8 \pi^{2} I c
$$

with a reported value ${ }^{36}$ of $0.0584 \mathrm{~cm}^{-1}$. The change in equilibrium bond length induced by the laser field will modify the moment of inertia and hence the rotational constant of the dimer. Substituting data from previous studies ${ }^{35-37}$ into eqn (3.9), it is readily determined that an irradiance of $10^{16} \mathrm{~W} \mathrm{~m}^{-2}$ will cause the dimer bond to extend by $1.72 \mathrm{pm}$. It is important to note that, despite this bond length being measured between the centers of each molecule, the linear expansion will be almost entirely operative through extension of the N-H hydrogen bond. [The principle of laserinduced bond extension could be applied to individual bonds in either HCN molecule, but the polarizability of each $\mathrm{HCN}$ molecule is much larger than that of any individual atomic component; also the N-H intermolecular bond has a 
much lower force constant than the intramolecular bonds.] The change in equilibrium bond length can be applied to eqn (3.10), giving a new rotational constant of $0.0579 \mathrm{~cm}^{-1}-$ a difference of about $1 \%$. This is experimentally very significant, well above the bounds of error in microwave spectroscopic measurements. Taking account of the distribution of intensity across a typical laser beam, it is clear that the effect would be manifest in a broadening as well as a shift in spectral lines.

\subsection{Molecular Solids}

To apply the theory we consider a regular solid comprising close-packed, electrically neutral quasi-linear molecules. Focusing on one neighboring pair of molecules in detail, we consider specifically a pair of parallel, cylindrically symmetric molecules with a mutual separation vector $\mathbf{R}$ orthogonal to their 'long' molecular axes. Identifying $\mathbf{R}$ with the Z-axis and the molecular axis with the X-direction, and assuming the system is irradiated with plane-polarized light, we define the polarization vector of throughput radiation in cylindrical coordinates as $\mathbf{e}=\sin \phi \cos \theta \hat{\mathbf{i}}+\sin \phi \sin \theta \hat{\mathbf{j}}+$ $\cos \phi \hat{\mathbf{k}}$, where $\phi$ and $\theta$ are the angles made by $\mathbf{e}$ with $\mathbf{R}$ and the molecular axis, respectively. From (5), the laserinduced energy shift experienced by this pair is;

$$
\begin{aligned}
\Delta E(R)=\frac{I}{\varepsilon_{0} c} \operatorname{Re} & \left\{\sin ^{2} \phi \cos ^{2} \theta \alpha_{X X}^{A} V_{X X} \alpha_{X X}^{B}+\sin ^{2} \phi \sin ^{2} \theta \alpha_{Y Y}^{A} V_{Y Y} \alpha_{Y Y}^{B}\right. \\
+ & \left.\cos ^{2} \phi \alpha_{Z Z}^{A} V_{Z Z} \alpha_{Z Z}^{B}\right\} \cos (\mathbf{k} \cdot \mathbf{R})
\end{aligned}
$$

On differentiating (3.11) with respect to distance, after taking the leading terms in the Taylor series expansions of cos $k R, \sin k R$ and cos k.R (effecting a correction to the result given in ref. 21) we obtain the following expression for the force induced between the particles, $\mathbf{F}_{\text {ind }}=-\partial \Delta E_{\text {ind }} / \partial \mathbf{R}$;

$$
\mathbf{F}_{\text {ind }}(R)=\frac{3 I}{4 \pi \varepsilon_{0}^{2} c R^{4}}\left[\alpha_{\perp}^{A} \alpha_{\perp}^{B}\left(\sin ^{2} \phi\left(2+\sin ^{2} \theta\right)-2\right)+\alpha_{\|}^{A} \alpha_{\|}^{B} \sin ^{2} \phi \cos ^{2} \theta\right],
$$

where $\alpha_{\perp}=\alpha_{Y Y}=\alpha_{Z Z}, \alpha_{\|}=\alpha_{X X}$. This is effectively the only mechanically operative pair force, since other interparticle forces in the solid are balanced at equilibrium. It is instructive to consider special geometric cases.

\section{Parallel pair}

The following results emerge for cases in which the polarization vector of the incident light is: $(i)$ parallel to the molecular axis; (ii) parallel to the separation vector, (iii) orthogonal to both;

$$
\mathbf{F}_{\text {ind }}(R)=\left\{\begin{array}{rr}
\frac{3 I\left(\alpha_{\|}^{A} \alpha_{\|}^{B}\right)}{4 \pi \varepsilon_{0}^{2} c R^{4}}, & (\text { i })(\phi=\pi / 2, \theta=0) \\
-\frac{3 I\left(\alpha_{\perp}^{A} \alpha_{\perp}^{B}\right)}{2 \pi \varepsilon_{0}^{2} c R^{4}}, & (\text { ii })(\phi=0, \theta=\pi / 2) \\
\frac{3 I\left(\alpha_{\perp}^{A} \alpha_{\perp}^{B}\right)}{4 \pi \varepsilon_{0}^{2} c R^{4}}, & \text { (iii) }(\phi=0, \theta=0)
\end{array}\right.
$$

The positive sign for results $($ i) and (iii) signifies a repulsion tending to increase the pair separation, leading to expansion; in case (ii) the attractive force will generate a contraction. The strengths of the forces depends on the magnitudes of $\alpha_{\|}^{\xi}$ and $\alpha_{\perp}^{\xi}$, and for electronically prolate molecules, $\left(\alpha_{\|}^{\xi}>\alpha_{\perp}^{\xi}\right)$, the repulsion $(i)$ is the largest force. 


\section{End-to-end pair}

The above results account for only two dimensions of molecular packing in these anisotropic solids; it is also necessary to consider the forces on a pair of adjacent particles placed end-to-end. Again the results differ according to whether the polarization vector of the incident radiation is: $(v)$ parallel to, or $(v i)$ orthogonal to the intermolecular separation vector. Explicitly, the ensuing forces are as follows;

$$
\mathbf{F}_{\text {ind }}^{0}(R)= \begin{cases}-\frac{3 I\left(\alpha_{\|}^{A} \alpha_{\|}^{B}\right)}{2 \pi \varepsilon_{0}^{2} c R^{4}}, & (v)(\mathbf{e} \| \mathbf{R}) \\ \frac{3 I\left(\alpha_{\perp}^{A} \alpha_{\perp}^{B}\right)}{4 \pi \varepsilon_{0}^{2} c R^{4}}, & (v i)(\mathbf{e} \perp \mathbf{R})\end{cases}
$$

Thus, irradiating such a solid with plane-polarized light effects a contraction in the direction of the solid parallel to the polarization of the laser beam, and an expansion in the other two orthogonal dimensions, both linearly dependent on the irradiance of the laser.

In summary, a compression parallel to e together with an expansion in directions perpendicular to e produces a solid deformation characterized by an overall increase in volume that scales linearly with the irradiance $I$. This in turn signifies a change in the local density - and hence in the local refractive index - that can also be expected to scale linearly with $I$. Optical electrostriction thus leads to an optomechanically induced intensity-dependence in the refractive index.

\section{DISCUSSION}

Detection of the process of optical electrostriction is fraught with experimental difficulty, and this is undoubtedly why it has not been characterized before. Such difficulties should not prove insurmountable, but they need to be taken into account in planning practicable experiments. Because the effect of interest leads to optical changes, there is an obvious temptation to seek an optical proof. However it is clear that the optical electrostrictive effect, in solids, will usually be dominated by much more widely known electronic mechanisms such as the optical Kerr effect ${ }^{38}$ - and, in absorbing regions, other photothermal effects due to spatially inhomogeneous heating. Intriguingly, electrostriction was indeed considered in connection with an intensity-dependent refractive index in the early days of laser physics, ${ }^{39}$ but it subsequently emerged that other mechanisms play a more direct and significant role.

With the problems envisaged with optical means of detecting this effect, consideration of mechanical methods may be met with more success. As seen in the previous section, the relatively modest change in inter-particle separation is magnified when considering a molecular solid transparent to the applied radiation field. Although the induced force and corresponding separation modification will be limited orthogonal to the propagation direction due to the width of the laser beam, if the radiation field propagates along the length of the solid, a much greater effect can be expected. This will result in an expansion of the material parallel to the propagation direction of the beam, since this is necessarily perpendicular to its polarization. A system that seems particularly suited to measure small changes in size is one recently decribed ${ }^{40}$ - the pertinent part being two mirrors set up opposite each other, of known separation such that a light wave propagating between the two has a known interference pattern. In their system the activation of a mechanical switch causes the position of one of the mirrors to shift slightly, altering the interference pattern. If instead the mirror were attached to a thin rod of material transparent to a given laser frequency, an intense laser pulse along the length of this rod would cause it to expand, altering the position of the mirror resulting in the modification of the interference pattern, the detection and quantification of which would allow the optical electrostriction effect to be detailed.

Although we have focused on the intrinsic interest of the subject, it is worth considering the applicability of this effect to optomechanical devices. In the context of dramatically accelerating developments in the field of nanoelectromechanical systems, any mechanism that can reproducibly deliver a reversible and ultrafast mechanical 
response, actuated by light, appears to be of considerable merit. It is hoped the mechanism described herein will provide many opportunities and challenges in this burgeoning field.

\section{REFERENCES}

1. D. O'Dell, S. Giovanazzi, G. Kurizki and V. M. Akulin, "Bose-Einstein condensates with 1/r interatomic attraction: Electromagnetically induced "gravity"', Phys. Rev. Lett., 84, pp. 5687-5690, 2000.

2. S. Giovanazzi, D. O'Dell and G. Kurizki, "One-dimensional compression of Bose-Einstein condensates by laser-induced dipole-dipole interactions", J. Phys. B: At. Mol. Opt. Phys., 34, pp. 4757, 2001.

3. S. Giovanazzi, D. O'Dell and G. Kurizki, "Self-binding transition in Bose condensates with laser-induced "gravitation"', Phys. Rev. A, 6303, 2001.

4. S. Giovanazzi, D. O'Dell and G. Kurizki, "Density modulations of Bose-Einstein condensates via laser-induced interactions", Phys. Rev. Lett., 88, 2002.

5. I. E. Mazets, D. H. J. O'Dell, G. Kurizki, N. Davidson and W. P. Schleich, "Depletion of a Bose-Einstein condensate by laser-induced dipole-dipole interactions", J. Phys. B: At. Mol. Opt., 37, pp. S155-S164, 2004.

6. M. Nieto-Vesperinas, P. C. Chaumet and A. Rahmani, "Near-field photonic forces (vol 362, pg 719, 2004)", Philos. Trans. R. Soc. Lond. Ser. A-Math. Phys. Eng. Sci., 362, pp. 2889-2890, 2004.

7. A. Salam, "A general formula for the rate of resonant transfer of energy between two electric multipole moments of arbitrary order using molecular quantum electrodynamics", J. Chem. Phys., 122, 044112, 2005.

8. A. Salam, "Resonant transfer of excitation between two molecules using Maxwell fields", J. Chem. Phys., 122, 044113, 2005.

9. J.-M. Fournier, J. Rohner, P. Jacquot, R. Johann, S. Mias and R.-P. Salathé, "Assembling mesoscopic particles by various optical schemes", Proc. SPIE, 5930, 59300Y, 2005.

10. A. Labeyrie, M. Guillon and J. M. Fournier, "Optics of laser trapped mirrors for large telescopes and hypertelescopes in space", Proc. SPIE, 5899, 58990V, 2005.

11. P. C. Chaumet and M. Nieto-Vesperinas, "Optical binding of particles with or without the presence of a flat dielectric surface", Phys. Rev. B, 6403, 035422, 2001.

12. S. K. Mohanty, J. T. Andrews and P. K. Gupta, "Optical binding between dielectric particles", Opt. Express, 12, pp. 2746-2753, 2004.

13. J.-M. R. Fournier, G. Boer, G. Delacretaz, P. M. Jacquot, J. Rohner and R. P. Salathé, "Building optical matter with binding and trapping forces", Proc. SPIE, 5514, pp. 309-317, 2004.

14. T. Thirunamachandran, "Intermolecular Interactions in the Presence of an Intense Radiation-Field", Mol. Phys., 40, pp. 393-399, 1980.

15. D. L. Andrews and M. J. Harlow, "Phased and Boltzmann-Weighted Rotational Averages", Phys. Rev. A, 29, pp. 2796-2806, 1984.

16. M. M. Burns, J. M. Fournier and J. A. Golovchenko, "Optical Binding", Phys. Rev. Lett., 63, pp. 1233-1236, 1989.

17. P. W. Milonni and M. L. Shih, "Source Theory of the Casimir Force", Phys. Rev. A, 45, pp. 4241-4253, 1992.

18. F. Depasse and J. M. Vigoureux, "Optical Binding Force between 2 Rayleigh Particles", J. Phys. D-Appl. Phys., 27, pp. 914-919, 1994.

19. J.-M. R. Fournier, M. M. Burns and J. A. Golovchenko, "Writing diffractive structures by optical trapping", Proc. SPIE, 2406, pp. 101-111, 1995.

20. P. W. Milonni and A. Smith, "van der Waals dispersion forces in electromagnetic fields", Phys. Rev. A, 53, pp. 3484-3489, 1996.

21. D. S. Bradshaw and D. L. Andrews, "Optically induced forces and torques: Interactions between nanoparticles in a laser beam", Phys. Rev. A, 72, 033816, 2005.

22. D. L. Andrews and D. S. Bradshaw, "Laser-induced forces between carbon nanotubes", Opt. Lett., 30, pp. 783 785, 2005.

23. D. S. Bradshaw and D. L. Andrews, "Interactions between spherical nanoparticles optically trapped in Laguerre-Gaussian modes", Opt. Lett., 30, pp. 3039-3041, 2005.

24. D. L. Andrews, R. G. Crisp and D. S. Bradshaw, "Optically induced inter-particle forces. From the bonding of dimers to optical electrostriction in molecular solids", J. Phys. B: At. Mol. Opt., (in press). 
25. E. A. Power and T. Thirunamachandran, "Analysis of the causal behavior in energy transfer between atoms", Phys. Rev. A, 56, 3395, 1997

26. E. A. Power and S. Zienau, "Coulomb Gauge in Non-Relativistic Quantum Electro-Dynamics and the Shape of Spectral Lines", Phil. Trans. Roy. Soc. Lond. Se. A - Math. Phys. Sci., 251, pp. 427-454, 1959

27. R. G. Woolley, "Molecular Quantum Electrodynamics", Proc. R. Soc. London Ser. A-Math. Phys. Eng. Sci., 321, pp. 557-572, 1971

28. M. Babiker, E. A. Power and T. Thirunamachandran, "On a Generalization of the Power-Zienau-Woolley Transformation in Quantum Electrodynamics and Atomic Field Equations", Proc. R. Soc. London Ser. AMath. Phys. Eng. Sci., 338, pp. 235-249, 1974

29. D. L. Andrews and D. S. Bradshaw, "Virtual photons, dipole fields and energy transfer: a quantum electrodynamical approach", Eur. J. Phys., 25, pp. 845-858, 2004

30. R. D. Jenkins, D. L. Andrews and L. C. Dávila Romero, "A new diagrammatic methodology for nonrelativistic quantum electrodynamics", J. Phys. B: At. Mol. Opt. Phys., 35, pp. 445-468, 2002

31. G. J. Daniels, R. D. Jenkins, D. S. Bradshaw and D. L. Andrews, "Resonance energy transfer: The unified theory revisited", J. Chem. Phys., 119, pp. 2264-2274, 2003

32. P. W. Milonni, "Field Quantization and Radiative Processes in Dispersive Dielectric Media", J. Mod. Opt., 42, pp. 1991-2004, 1995

33. G. Juzeliunas, "Microscopic theory of quantization of radiation in molecular dielectrics: Normal-mode representation of operators for local and averaged (macroscopic) fields ", Phys. Rev. A, 53, pp. 3543-3558, 1996

34. G. T. Gao, J. B. Woller, X. C. Zeng and W. C. Wang, "Vapour - liquid equilibria of binary mixtures containing Stockmayer molecules ", J. Phys.-Condes. Matter, 9, pp. 3349-3360, 1997

35. S. V. Churakov and M. Gottschalk, Geo. Cos. Acta, 67, pp. 2397, 2002

36. K. Somasundram, R. D. Amos and N. C. Handy, Theor. Chim. Acta, 69, pp. 491, 1986

37. C. M. Dion, A. Keller, O. Atabek and A. D. Bandrauk, "Laser-induced alignment dynamics of HCN: Roles of the permanent dipole moment and the polarizability ", Phys. Rev. A, 59, pp. 1382-1391, 1999

38. A. D. Buckingham, "Birefringence Resulting from the Application of an Intense Beam of Light to an Isotropic Medium", Proceedings of the Physical Society of London Section B, 69, pp. 344-349, 1956.

39. R. Y. Chiao, E. Garmire and C. H. Townes, "Self-Trapping of Optical Beams", Phys. Rev. Lett., 13, pp. 479482, 1964.

40. Z. Xie and H. F. Taylor, "Fabry-Perot optical binary switch for aircraft applications", Opt. Lett., 31, pp. 26952697,2006 